\title{
Waveguide CARS Spectroscopy: A New Method for Background Suppression, Using Dielectric Layers as a Model
}

\author{
WIM P. de BOEIJ, HANS S. KANGER, GERALD W. LUCASSEN, \\ CEES OTTO and JAN GREVE* \\ Applied Optics Group, Department of Applied Physics, University of Twente, P.O. Box 217, 7500 AE Enschede, The Netherlands
}

\begin{abstract}
Waveguide Coherent Anti-Stokes Raman Spectroscopy (CARS) can be used to measure Raman-active vibrations in thin-layer dielectric waveguides. In waveguide CARS experiments, background-free spectra can be obtained when asymmetric mode combinations are applied. The degree of suppression depends on the waveguide parameters and the wavelengths used. A new method using scanning pump and Stokes beams in waveguide CARS experiments is presented, which allows the possibility of maintaining full background suppression conditions over large spectral intervals. A small controlled change in the tuning conditions results in a heterodyning of the signal with a small amount of background, thereby enhancing the small resonant signals. Several simulations for dielectric waveguides are given.
\end{abstract}

Index Headings: Background suppression; Nonlinear spectroscopy; Raman spectroscopy; Waveguides.

\section{INTRODUCTION}

The combination of Raman spectroscopic techniques with integrated waveguide optics yields a useful tool for the determination and identification of structure and orientation in thin films. ${ }^{1,2}$ Like Spontaneous Raman Spectroscopy, Surface (Waveguide) Coherent Raman Spectroscopy (CARS or CSRS) can be used to probe Raman-active vibrations in thin-layer waveguide structures. ${ }^{3}$ Due to the large electrical field strengths present in optical waveguides, strong nonlinear optical effects are induced, and intense CARS signals are expected. ${ }^{4}$ Surface CARS enables direct, nondestructive measurement of dielectric film characteristics and has been applied successfully to films based on polymer materials. ${ }^{3}$ It has been shown that low concentrations of additives in polymer films based on PMMA can be measured by surface CARS. ${ }^{5}$ CARS spectroscopy in condensed matter is often hampered by the presence of a strong nonlinear nonresonant background, which interferes with the Raman vibrations, yielding complicated spectra. Although background suppression techniques like polarizationsensitive CARS have excellent suppression qualities when applied in bulk media, ${ }^{6,7}$ they are not applicable in waveguide structures due to the discrete polarization orientations of the guided modes. In surface CARS spectroscopy, however, the background can be fully eliminated by applying the appropriate mode combinations for pump and Stokes fields and selecting the correct CARS signal mode. ${ }^{4}$ The background suppression results from a destructive interference effect between the different signal contributions inside the film, substrate, and cladding. If background contributions of film and substrate are fully suppressed, very high sensitivities can be achieved. ${ }^{6,7}$ With

\footnotetext{
Received 26 October 1992.

* Author to whom correspondence should be sent.
}

this suppression technique, spectra of thin films composed of a few monomolecular overlayers have been measured. ${ }^{8-12}$

Although in principle full background suppression is possible, the optimum suppression occurs only at one spectral shift. Due to the spectral dependence of the mode structures and refractive indices, good background suppression is limited to only a narrow spectral range. In this article we discuss a technique to increase the spectral range where full background suppression is possible. The technique is based on the fact that a change in the pump wavelength (while the shift is kept constant) results in a change in the position of the suppression minimum. Therefore each spectral shift has its own optimum pump (and Stokes) wavelength, where full background suppression is possible. The organization of the article is the following. In the theoretical section the nonlinear waveguide theory is given, followed by an experimental section where spectra of a polystyrene dielectric waveguide are presented. The suppression method is explained, and results of calculations on dielectric waveguides are presented and discussed.

\section{THEORY}

The theory of dielectric optical waveguides is discussed in detail by Marcuse. ${ }^{13}$ In Fig. 1 the geometry of a threelayer waveguide structure is shown. Waveguide structures can only contain a limited number of guided waves, with two distinct polarization conditions-TM modes which are polarized perpendicular to the $y$-direction, and TE modes which are polarized parallel to the y-direction. The electric field for the TE polarized wave with frequency $\omega_{p}$ can be expressed as:

$$
\vec{E}_{p}^{k}=1 / 2 \vec{e}_{\mathrm{y}} e^{\left(j\left(\omega_{p} t-\beta_{p}^{k} x\right)\right)} f_{p}^{k}(\mathrm{z}) a_{p}^{k}(\mathrm{x})+\text { c.c. }
$$

where $\omega_{p}$ denotes the frequency, $\beta_{p}^{k}$ denotes the propagation vector of the mode, $f_{p}^{k}(\mathrm{z})$ denotes the electric field distribution function, $a_{p}^{k}(\mathrm{x})$ denotes the amplitude distribution function, c.c. denotes the complex conjugate, and $k$ enumerates the different modes.

The electric field distribution $f_{p}^{k}(\mathrm{z})$ for the different modes as well as the propagation vector $\beta_{p}^{k}$ can be calculated by solving an $n$-media dispersion relation for the electromagnetic waves propagating in isotropic layers, ${ }^{14}$ or by the matrix method given by Ghatak et al. ${ }^{15}$

The theory of third-order nonlinear processes in thinlayer waveguides has been given by several authors. ${ }^{16,17}$ For third-order nonlinear optical processes such as CARS, the signal is generated by a nonlinear polarization $\vec{P}^{(3)}$ given by: 18 


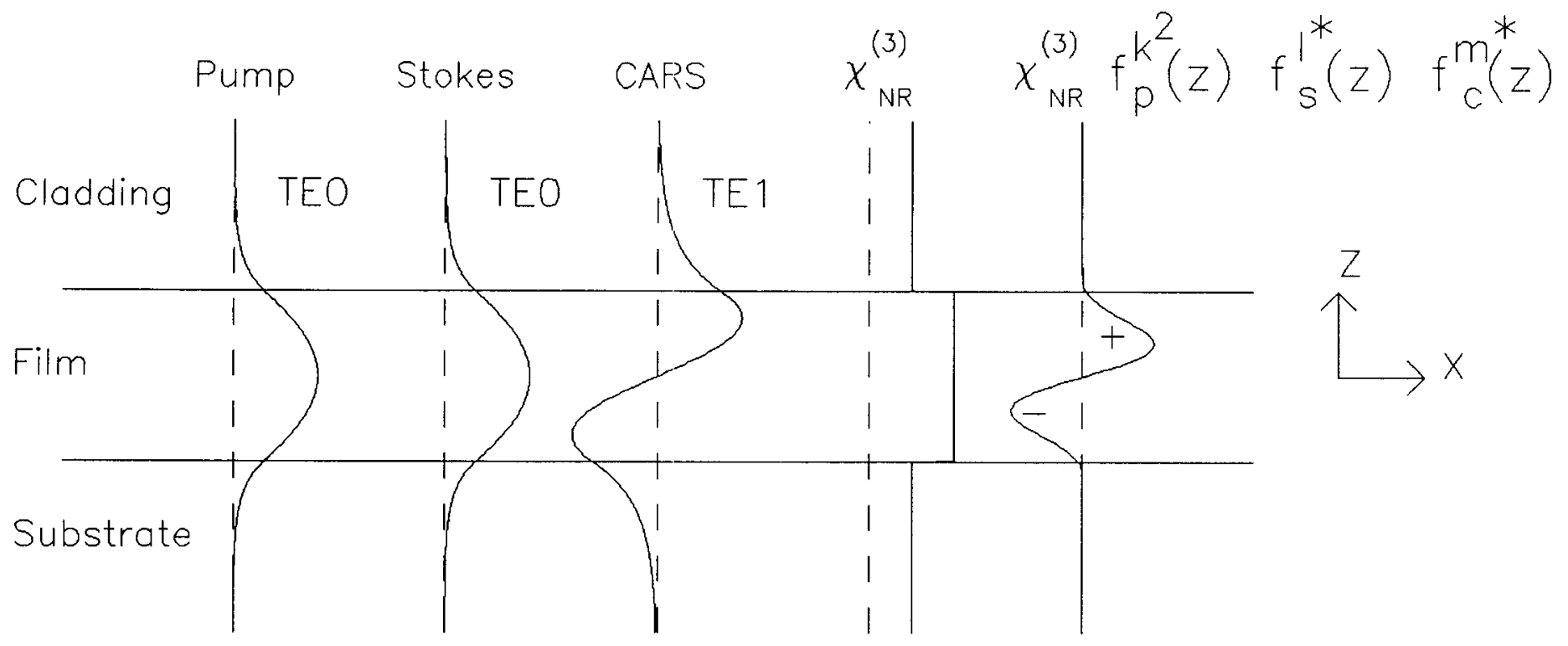

Fig. 1. Three-layer dielectric waveguide, consisting of cladding, film, and substrate. The electrical field distributions for the two lowest-order TE polarized guided waves are shown (TE0, TE1). The definitions of the coordinate-axis are given in the inset. The nonresonant $\chi^{(3)}$ distribution and the projection of the generated CARS polarization on the CARS mode are indicated as well.

$$
\begin{aligned}
\vec{P}^{(3)}= & \overline{\bar{\chi}}^{(3)}\left(\omega_{\text {CARS }} ; \omega_{\text {probe }}, \omega_{\text {pump }},-\omega_{\text {Stokes }}\right): \\
& \vec{E}_{\text {pump }} \cdot \vec{E}_{\text {probe }} \cdot \vec{E}_{\text {Stokes }}^{*}
\end{aligned}
$$

with

$$
\omega_{\text {CARS }}=\omega_{\text {probe }}+\omega_{\text {pump }}-\omega_{\text {Stokes }} .
$$

In degenerate CARS, $\omega_{\text {pump }}$ and $\omega_{\text {probe }}$ are equal, and the polarization $\vec{P}^{(3)}$ reduces to:

$$
\vec{P}^{(3)}=\overline{\bar{\chi}}^{(3)}\left(\omega_{c} ; \omega_{p}, \omega_{p},-\omega_{s}\right): \vec{E}_{p} \cdot \vec{E}_{p} \cdot \vec{E}_{s}^{*}
$$

with

$$
\omega_{c}=2 \omega_{p}-\omega_{s}
$$

and where the subscript $c$ denotes the CARS field, $p$ the pump, and $s$ the Stokes field.

If the waveguide consists of isotropic layers, the $\overline{\bar{\chi}}^{(3)}$ tensor consists of only 21 nonzero elements, of which three are independent:

$$
\chi_{1111}^{(3)}=\chi_{1221}^{(3)}+\chi_{1212}^{(3)}+\chi_{1122}^{(3)} .
$$

If one assumes only $\mathrm{TE}$ polarized waves for the pump and Stokes fields, the third-order induced polarization $\vec{P}^{(3)}(\mathrm{z}, \mathrm{x})$ present in the medium is given by:

$$
\begin{aligned}
\vec{P}^{(3)}(\mathrm{z}, \mathrm{x})= & \overline{\bar{\chi}}^{(3)}(\mathrm{z}):\left\{\vec{E}_{0, p}^{k}(\mathrm{z})\right\}^{2}\left\{\vec{E}_{0, s}^{l}(\mathrm{z})\right\}^{*} \\
& \cdot\left\{a_{p}^{k}(\mathrm{x})\right\}^{2}\left\{a_{s}^{l}(\mathrm{x})\right\}^{*} e^{-j\left(2 \beta_{p}^{k}-\beta_{s}^{l}\right) x}
\end{aligned}
$$

with

$$
\begin{aligned}
& \vec{E}_{0, p}^{k}(\mathrm{z})=1 / 2 \vec{e}_{\mathrm{y}} e^{j\left(\omega_{p} t\right)} f_{p}^{k}(\mathrm{z}) \\
& \vec{E}_{0, \mathrm{~s}}^{l}(\mathrm{z})=1 / 2 \vec{e}_{\mathrm{y}} e^{j\left(\omega_{s} t\right)} f_{s}^{l}(\mathrm{z}) .
\end{aligned}
$$

If phase matching is assumed (e.g., $\beta_{c}^{m}=2 \beta_{p}^{k}-\beta_{s}^{1}$ ), and $\beta_{c}^{m}$ is a possible guided wave solution for a mode with frequency $\omega_{c}$, the induced $\vec{P}^{(3)}$ generates a mode which propagates along the waveguide. Solving the problem of finding the intensity in guided modes needs the application of coupled mode theory. For TE modes the ex- pression for the amplitude dependence on $\mathrm{x}$ for the $m$ th CARS mode is given by: $\dagger$

$\frac{\mathrm{d}}{\mathrm{dx}}\left(a_{c}^{m}\right)=-\frac{j \omega}{4} e^{j \beta_{c}^{m} \mathrm{x}} \cdot \int_{-\infty}^{+\infty} \vec{P}^{(3)}(\mathrm{z}, \mathrm{x}) \cdot\left\{\vec{E}_{0, c}^{m}(\mathrm{z})\right\}^{*} \mathrm{dz}$.

Substitution of the induced polarization $\vec{P}^{(3)}(\mathrm{z}, \mathrm{x})$, yields:

$$
\frac{\mathrm{d}}{\mathrm{dx}}\left(a_{c}^{m}\right)=-\frac{j \omega}{4} e^{-j\left(2 \beta_{p}^{k}-\beta_{s}^{l}-\beta_{c}^{m}\right) \mathrm{x}}\left\{a_{p}^{k}(\mathrm{x})\right\}^{2}\left\{a_{s}^{l}(\mathrm{x})\right\}^{*} F^{k l m}
$$

where $F^{k l m}$, the overlap integral, is given by:

$$
\begin{gathered}
F^{k l m}=\int_{-\infty}^{+\infty} \bar{\chi}^{(3)}(\mathrm{z}): \vec{E}_{0, p}^{k}(\mathrm{z}) \vec{E}_{0, p}^{k}(\mathrm{z})\left\{\vec{E}_{0, s}^{l}(\mathrm{z})\right\}^{*} \\
\cdot\left\{\vec{E}_{0, c}^{m}(\mathrm{z})\right\}^{*} \mathrm{dz} .
\end{gathered}
$$

For an induced polarization $\vec{P}^{(3)}$ between $\mathrm{x}=0$ and $\mathrm{x}=$ $L$, the expression of the amplitude $a_{c}^{m}$ of the generated mode $m$ can be found by integrating from $\mathrm{x}=0$ to $\mathrm{x}=L$ :

$$
a_{c}^{m}=-\frac{j \omega}{4}\left(a_{p}^{k}\right)^{2}\left\{a_{s}^{l}\right\}^{*} F^{k l m} \int_{0}^{L} e^{-j\left(2 \beta_{p}^{k}-\beta_{s}^{l}-\beta_{c}^{m}\right) \mathrm{x}} \mathrm{dx} .
$$

Solving the integral gives:

$$
a_{c}^{m}=\frac{j \omega}{4}\left(a_{p}^{k}\right)^{2}\left\{a_{s}^{l}\right\}^{*} F^{k l m}(j \Delta \beta)^{-1}\left\{e^{-j \Delta \beta L}+1\right]
$$

with

$$
\Delta \beta=2 \beta_{p}^{k}-\beta_{s}^{l}-\beta_{c}^{m}, \quad \text { the phase mismatch. }
$$

With the expression for the amplitude of the $m$ th CARS mode, it is straightforward to find the power $\mathcal{P}$ of that specific mode. The electrical fields $\vec{E}_{p}^{k}$ are normalized in such a way that $|a|^{2}$ gives the guided wave power per meter wavefront (in the $y$-direction) in units of $\mathrm{W} / \mathrm{m}$. When all waves have a width $H$ we find:

† See Ref. 16. According to our calculations, the electric field $E$ should be replaced by its complex conjugate, $E^{*}$. 


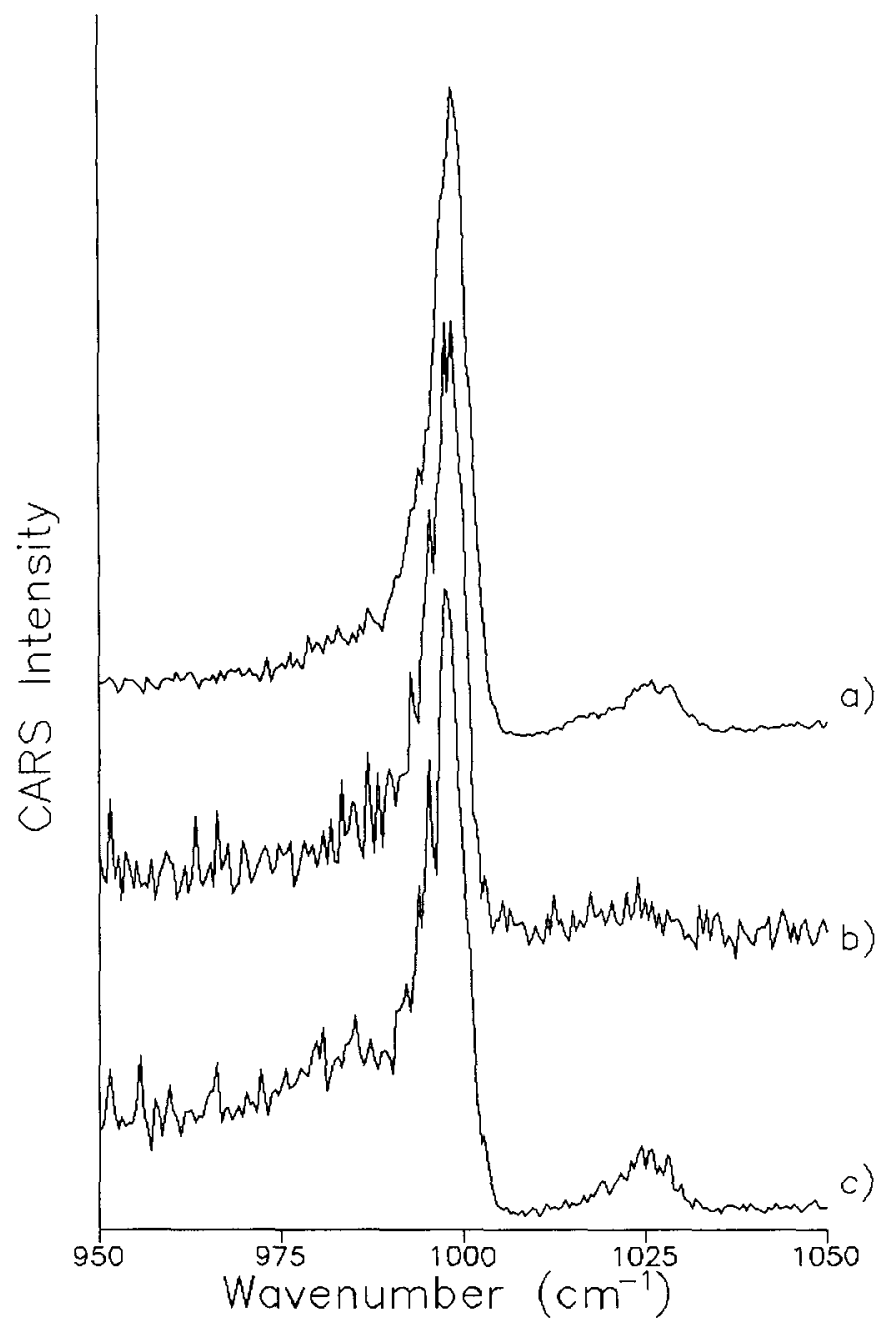

Fig. 2. Waveguide CARS spectrum of polystyrene. (a) TE000 mode combination. (b) TM000 mode combination. The layer thickness is 1.0 $\mu \mathrm{m}$. (c) CARS spectrum of bulk polystyrene material.

$$
\left|a_{p}^{k}\right|^{2} H=\mathcal{P}_{p}^{k} ;\left|a_{s}^{l}\right|^{2} H=\mathcal{P}_{s}^{l} ;\left|a_{c}^{m}(L)\right|^{2} H=\mathcal{P}_{c}^{m} .
$$

Combining Eqs. 14 and 16, the CARS signal power $\mathcal{P}_{c}^{m}$ is now given by:

$$
\mathcal{P}_{c}^{m}=\frac{\omega^{2}}{16}\left[\frac{L}{H}\right]^{2}\left(\mathcal{P}_{p}^{k}\right)^{2} \mathcal{P}_{s}^{l}\left|F^{k l m}\right|^{2} \operatorname{sinc}^{2}\left(\frac{\Delta \beta L}{2}\right) .
$$

For the TM modes exactly the same expression is found for the CARS signal power $\mathcal{P}_{c}^{m}$, with $F^{k l m}$ also given by Eq. 12.

\section{EXPERIMENTAL}

The second harmonic of a Nd:YAG laser (Quanta-Ray DCR-2, operated at $10 \mathrm{~Hz}$, pulse duration $8 \mathrm{~ns}$ ), was used for the pump beam at $532 \mathrm{~nm}$. Part of the second harmonic was used to pump a Quanta-Ray PDL-2 dye laser (Rhodamine 6G), which provided the tunable Stokes beam. Polarization directions of the beams were adjusted by Glan-Taylor polarizers. The generated CARS signal was analyzed by a Glan-Taylor polarizer and collected by a spherical mirror. The signal was focused onto the entrance slit of a double monochromator (UV-VIS 200 $\mathrm{mm}$, Jobin-Yvon) and detected by a photomultiplier (RCA 9973B). The computer and electronics were inter- faced by an IEEE bus. The CARS signals were digitized in an 8-channel 12-bit ADC and stored on floppy disk for later analysis. The spectra were measured in a scanning mode with the pump frequency fixed and Stokes frequency tunable. Spectra were recorded over an interval of 950 to $1050 \mathrm{~cm}^{-1}$ in 100 points with 80 pulses averaged per point. All spectra shown were corrected for laser intensity variations.

Polystyrene was dissolved in toluene, and spin-coated on an optical crown glass substrate. The thickness was determined interferometrically, with a surface profiler, and determined to be $1.2 \mu \mathrm{m}$. Two SF-6 coupling prisms (separated by $1 \mathrm{~cm}$ ) were used to couple the pump and Stokes beams in, and the CARS signal out, of the polystyrene waveguide. Pump and Stokes beams were applied under a small angle (1-2 degrees) to ensure phase matching. The effective overlap length was thereby reduced to $2 \mathrm{~mm}$, with beam widths in the order of 0.5 $\mathrm{mm}$. Figure $2 \mathrm{a}$ and $2 \mathrm{~b}$ show the waveguide CARS spectra of polystyrene for different polarization conditions. Figure $2 \mathrm{a}$ shows the results for all fields in the TEO mode. Figure $2 b$ gives the results for all fields in the TMO mode. For a comparison, the bulk spectrum of a thin polystyrene sample is given in Fig. 2c.

\section{WAVEGUIDE BACKGROUND SUPPRESSION}

In the expression for the CARS signal intensity, the factor $F^{k l m}$ contains the different mode distributions for pump, Stokes, and CARS fields as well as the third-order nonlinear susceptibilities of each layer. The nonlinear susceptibility $\overline{\bar{\chi}}^{(3)}$ can be written as:

$$
\overline{\bar{\chi}}=\overline{\bar{\chi}}^{\mathrm{NR}}+\overline{\bar{\chi}}^{\mathrm{R}} \text {, }
$$

where the superscript (3) is dropped and where $\overline{\bar{\chi}}^{\mathrm{NR}}$ is the nonresonant term, containing all nonresonant contributions, whereas $\overline{\bar{\chi}}^{R}$ contains all Raman vibrational resonances. CARS spectroscopy is often hampered by the presence of the nonlinear background, which interferes with the Raman lines. Due to the discrete polarization direction of the guided modes (TE or TM), polarization techniques ${ }^{6,7}$ fail to reduce this background. Only pulse sequenced techniques ${ }^{19}$ might give a reasonable reduction of the background. However, waveguide CARS provides a new method to suppress the background. The nature of the $F^{k l m}$ term can be expressed as a projection of the induced polarization term $\left\{f_{p}^{k}(\mathrm{z})\right\}^{2}$ $\left\{f_{s}^{l}(\mathrm{z})\right\}^{*}$ on the mode distribution function $\left\{f_{c}^{m}(\mathbf{z})\right\}^{*}$ weighted by $\overline{\bar{\chi}}^{(3)}(\mathrm{z})$, which can be taken as constant across a single layer. In case the Raman resonant vibrations are not taken into account (far away from any resonances), the above expression can be rewritten as:

$$
\begin{aligned}
F_{\mathrm{NR}}^{k l m}= & \int_{-\infty}^{0} \chi_{s u}^{\mathrm{NR}}\left\{f_{p}^{k}(\mathrm{z})\right\}^{2}\left\{f_{s}^{l}(\mathrm{z})\right\}^{*}\left\{f_{c}^{m}(\mathrm{z})\right\}^{*} \mathrm{dz} \\
& +\int_{0}^{h} \chi_{f i}^{\mathrm{NR}}\left\{f_{p}^{k}(\mathrm{z})\right\}^{2}\left\{f_{s}^{l}(\mathrm{z})\right\}^{*}\left\{f_{c}^{m}(\mathrm{z})\right\}^{*} \mathrm{dz} \\
& +\int_{h}^{\infty} \chi_{c l}^{\mathrm{NR}}\left\{f_{p}^{k}(\mathrm{z})\right\}^{2}\left\{f_{s}^{l}(\mathrm{z})\right\}^{*}\left\{f_{c}^{m}(\mathrm{z})\right\}^{*} \mathrm{dz}
\end{aligned}
$$

where the subscript " $c l$ " indicates cladding, " $f$ " the 


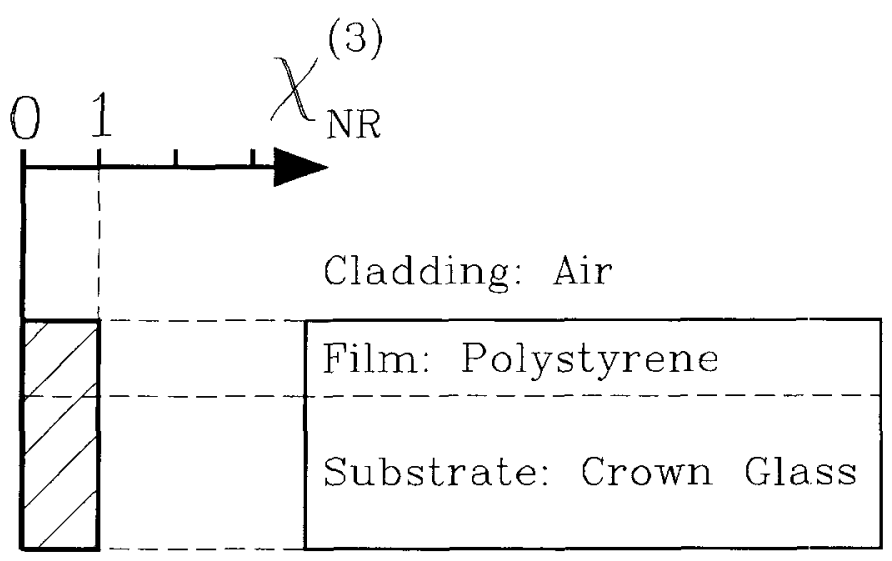

FIG. 3A. Waveguide structure as used in the simulations. The nonresonant susceptibility of each layer is indicated.

guiding film layer, and "su" the substrate. Full background suppression is obtained whenever the $F_{\mathrm{NR}}^{k l m}$ term is equal to zero. When all modes (pump, Stokes, and CARS) are chosen in the TEO mode, the mode combination is depicted by TE000 (TE-pSC, with $p$ the pump, $\mathrm{S}$ the Stokes, and $\mathrm{C}$ the CARS mode number). With the selection of an asymmetric mode combination for the pump, Stokes, and CARS mode (e.g., TE001, TE101, TE110, or TE010), the separate contributions from substrate, film, and cladding can cancel, as is depicted in Fig. 1 for the TE001 mode combination. Since the mode distribution functions $f_{p}^{k}$, $f_{s}^{l}$, and $f_{c}^{m}$ strongly depend on the frequency of the pump, Stokes, and CARS fields (given by the pump wavelength and spectral shift), the suppression is expected to change whenever one of these parameters is changed. The region of full suppression is therefore limited to a narrow spectral interval. A possible way to increase the spectral interval is to change the pump wavelength for different shifts. The three wavelengths are tuned for each spectral point to a position where the nonresonant background contribution is zero again. In the following section this method is demonstrated for a simple dielectric waveguide.

\section{RESULTS}

Simulations were performed on an air/polystyrene/glass waveguide. The structure of this waveguide is given in Fig. 3A. The dispersion of the refractive indices of the film layer and the substrate is indicated in Fig. 3B and is taken into account in the calculations. The nonresonant third-order susceptibility $\overline{\bar{\chi}}_{\mathrm{NR}}^{(3)}$ is assumed to be zero in air and has a value which is equal in the film and substrate. A computer program was developed to perform the simulations. After setting the parameters of the waveguide structure (number of layers, refractive indices, dispersion, and layer thickness), one selects the pump wavelength. With these parameters and the selected CARS shift, which determines both Stokes and CARS wavelength, all possible modes (both TE and TM) of this configuration can be calculated. For all mode combinations, the contribution to the CARS signal can be evaluated for each layer. The program has the possibility of determining the CARS signal dependence on several parameters, such as layer thickness, refractive index of each layer, pump wavelength, and spectral shift (CARS spectrum).

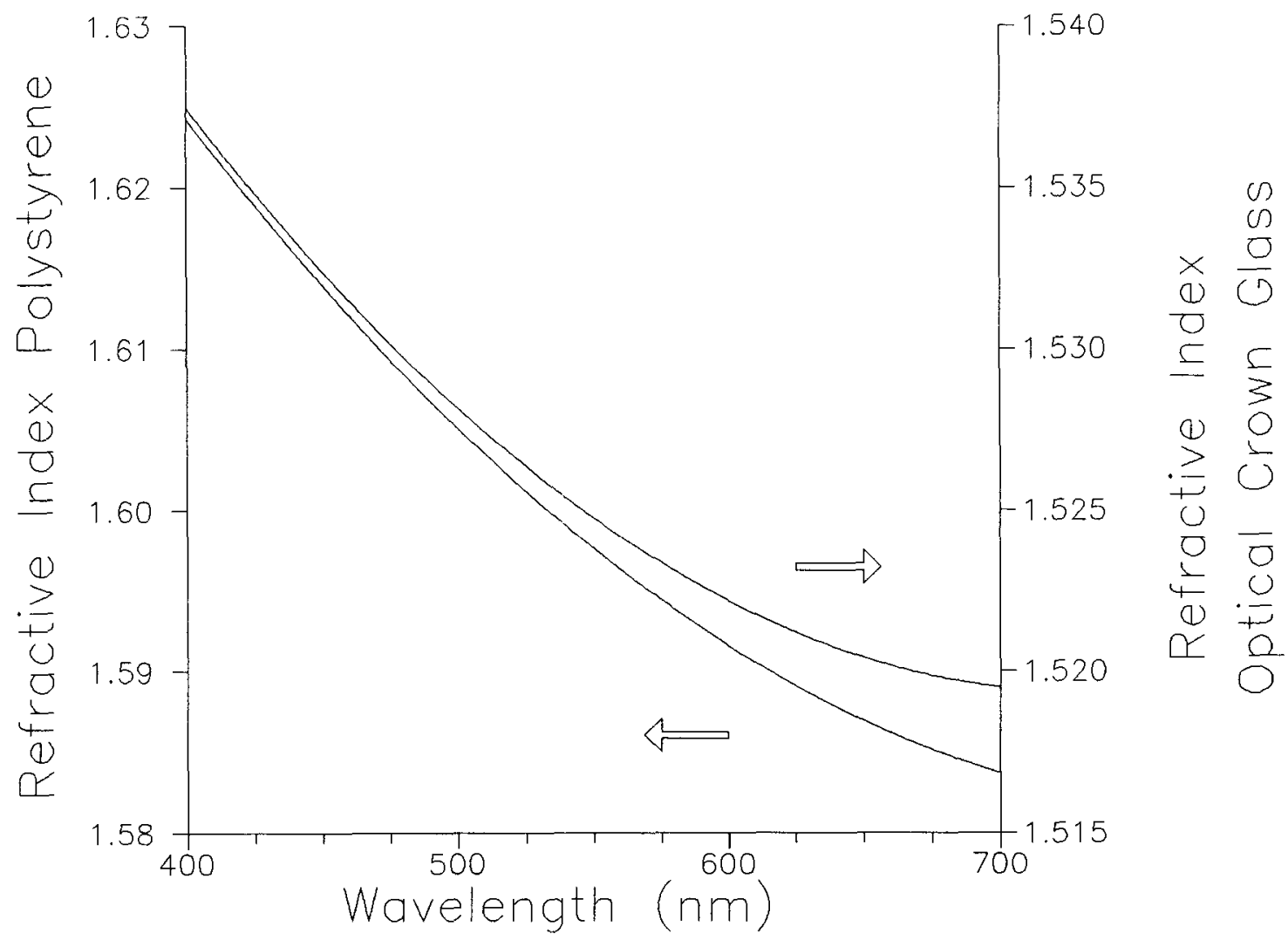

FIG. 3B. Dispersion of the refractive index of the polystyrene film layer and crown glass substrate. 


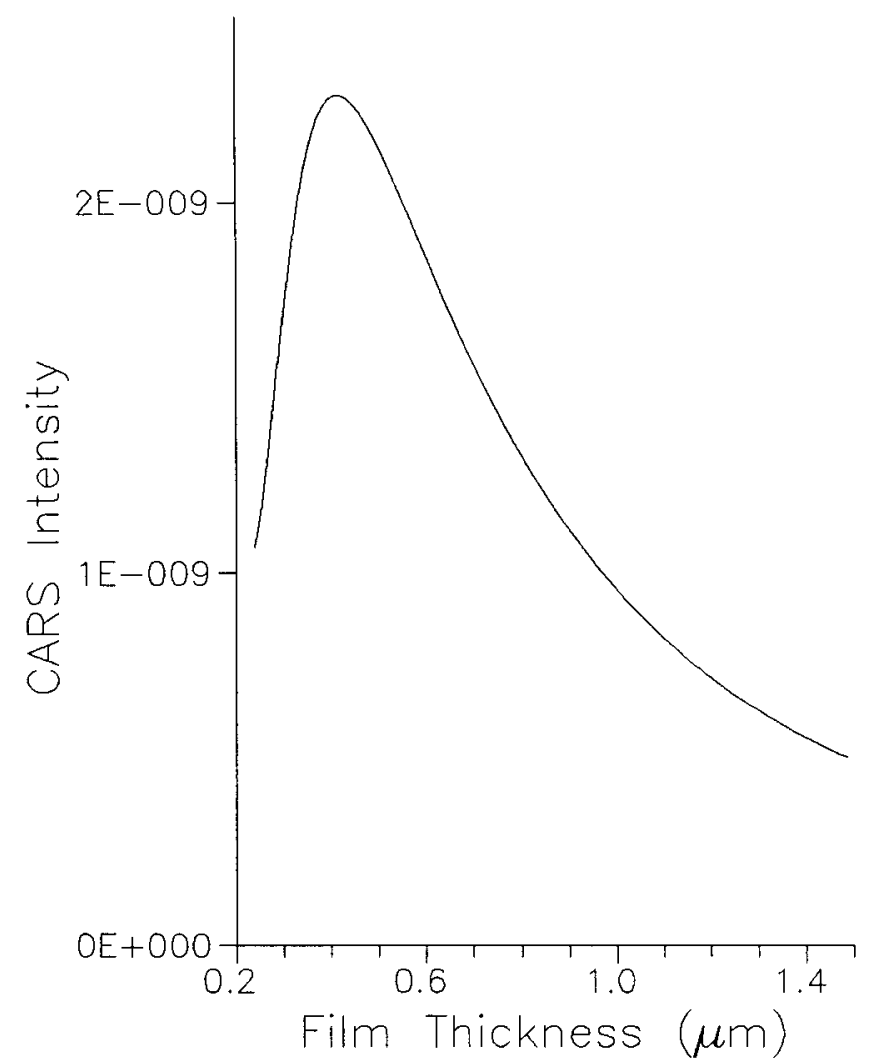

FIG. 4. Dependence of the nonresonant CARS contributions in the TE000 mode combination from the waveguide as a function of film layer thickness. The spectral shift was set to $1000 \mathrm{~cm}^{-1}$ and pump wavelength was set to $532 \mathrm{~nm}$.

In Fig. 4 the dependence of the nonresonant CARS contribution from the waveguide as a function of layer thickness is given. In the calculations the factor $\left[\frac{L}{H}\right]^{2}$ $\left(\mathcal{P}_{p}^{k}\right)^{2} \mathcal{P}_{s}^{l} \operatorname{sinc}^{2}\left(\frac{\Delta \beta L}{2}\right)$ in Eq. 17 was set to 1 , and calculations were done with a pump wavelength of $532 \mathrm{~nm}$ and a spectral shift of $1000 \mathrm{~cm}^{-1}$. All fields (pump, Stokes, and CARS) were chosen in the TE mode. The TE000 CARS signal dependence on the thickness, depicted in Fig. 4, resembles the results given in Ref. 4. The generation of signal in the TE000 mode shows a maximum at a film thickness of $0.42 \mu \mathrm{m}$. Due to the asymmetric waveguide configuration, no modes are possible below $0.25 \mu \mathrm{m}$. For thin film layers, the energy is distributed over all three layers, thereby exhibiting no pronounced maximum in the electric field distribution, resulting in a low nonlinear scattering. For thick film layers, however, the energy is fully guided by the film, but due to the large film thickness, no pronounced maximum in the electric field distribution is seen, which again gives a small nonlinear scattering. Since for each thickness the electrical fields are normalized in such a way that the guided power per meter wavefront is equal to $1 \mathrm{~W}$, the maximum in Fig. 4 results from an optimum between the film thickness and the energy content in the film layer. The sharper the electrical fields are peaked, the more efficient is the generation of CARS signals.

In Fig. 5 the CARS signal as a function of film layer thickness is given for different mode combinations. When examining the symmetric mode combinations in Fig. 5 [TEklm with $(1+m)$ even], we see that curves similar to those for the TE000 mode combination are obtained. The calculations were also done with the same parameters as in Fig. 4, a pump wavelength of $532 \mathrm{~nm}$ and a spectral shift of $1000 \mathrm{~cm}^{-1}$. The asymmetric mode combinations [TEklm with $(1+m)$ odd], however, show totally different behavior. Some of these mode combinations have sharp minima where no nonresonant CARS signal is expected (e.g., for the TE001 and TE101 mode combination), while other mode combinations (e.g., TE010 and TE110) show no minima in this range. The sharp minima are the result of a destructive interference between an equal contribution of the film layer and the cladding, which differ only in sign. For the asymmetric mode combinations which have no background suppression minimum, the two contributions from substrate and film do not cancel each other, independent of the layer thickness. When one is using a waveguide with a specific thickness and specific mode combination in a background-free regime, changes due to thin layers on top of this waveguide can be monitored. ${ }^{11}$ Due to the spectral dependence of refractive indices and mode distribution functions, the suppression thickness strongly depends on the spectral shift. In Fig. 5 two curves are shown for a shift of $1200 \mathrm{~cm}^{-1}$. For the TE001 mode combination a strong shift in the optimum thickness can be seen, whereas for the TE101 mode combination no minima are present. Due to this dependence, the ability to use the background suppression technique is limited to small spectral intervals. A waveguide with a thickness designed for optimum suppression conditions at $1000 \mathrm{~cm}^{-1}$ for a specific mode combination will therefore not work at other spectral shifts.

A possible way to overcome this problem is found in the tunability of the suppression minimum with the pump wavelength. This dependence was calculated and is given in Fig. 6. The calculations were done for a TE101 mode combination and film thickness of $1.0 \mu \mathrm{m}$. The Stokes and CARS wavelengths were calculated with the use of the applied pump wavelength and spectral shift. The curves shown in Fig. 6 are normal nonresonant CARS spectra. Three different pump wavelengths have been chosen, each having its own spectral shift where full background suppression is obtained. With an increase in the pump wavelength, the suppression minimum shifts towards the smaller wavenumbers. With the ability to tune the point of background suppression by changing the pump wavelength, it is now possible to select the pump wavelength for each spectral point in such a way that no background is measured along the whole spectral range. The pump tuning curves depend on waveguide configuration as well as mode combination. With these curves both Stokes and CARS signal frequencies can be calculated from pump wavelength and spectral shift, thereby taking into account the spectral shift dependence of the optimum pump wavelength.

The optimum pump tuning curves for two-mode combinations have been calculated and are given in Fig. 7 . Due to the steep dependence of the optimum pump wavelength for the TE001 mode combination as given in Fig. $7 \mathrm{~A}$, large pump changes are needed for maintaining the 


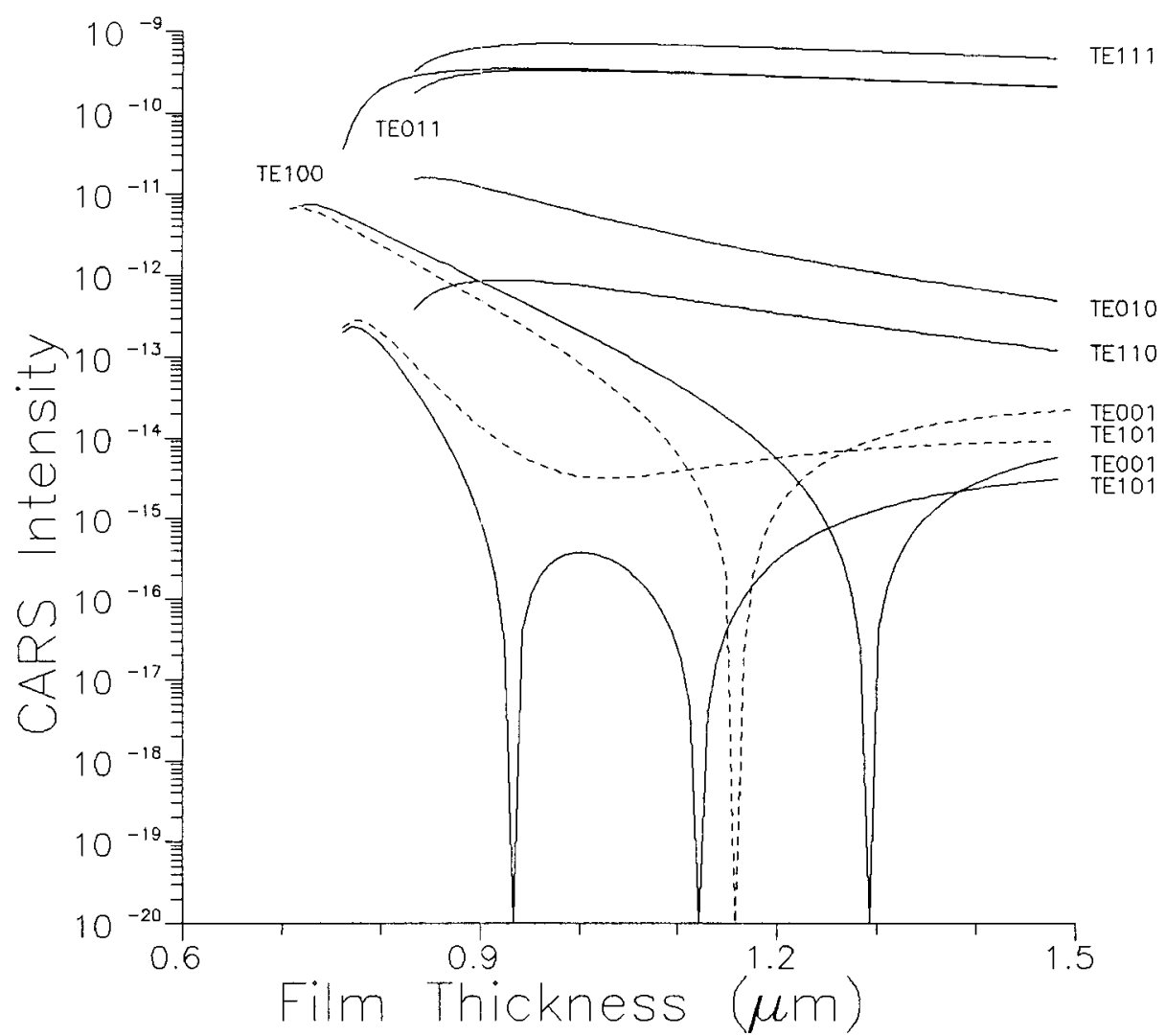

FIG. 5. Thickness dependence of the nonresonant CARS signal for different mode combinations. The solid line indicates a spectral shift of 1000 $\mathrm{cm}^{-1}$; the dashed line gives the results for the TE001 and TE101 mode combination at $1200 \mathrm{~cm}^{-1}$.

optimum conditions. Though possible in principle, in practice this approach results in serious experimental problems. Coupling angles need large adjustments during the spectral scan, with possible changes in the incoupling efficiencies. In case the excitation wavelengths approach electronic resonance conditions, large dispersion effects due to nonconstant CARS excitation profiles are expected. The best case for the pump tuning curve is a curve which needs minimal pump tuning during spectral scan. For the TE101 mode combination, a degenerate situation is observed in Fig. 7B. For one spectral shift, two possible pump wavelengths are possible. The upper part of the curve shows the best tuning properties. In this region, small pump tuning ranges can be used to cover a large spectral interval (e.g., with a waveguide thickness of $1.1 \mu \mathrm{m}$ ), and a 50-nm pump detuning range covers almost $400 \mathrm{~cm}^{-1}\left(800-1200 \mathrm{~cm}^{-1}\right)$.

The feasibility of background suppression in the application of thin overlayers was studied by the system shown in Fig. 8, which consists of a thin overlayer on top of a waveguide substrate combination. The crown glass substrate has a refractive index as given in Fig. $3 \mathrm{~B}$. The film layer has the same properties (refractive index and dispersion) as the polystyrene layer given in Fig. 3B. The refractive index of the overlayer is set at 1.4 and assumed to be wavelength independent. The nonresonant and resonant contributions of each layer are depicted in Fig. 8. The overlayer thickness was chosen to be $20 \mathrm{~nm}$ and the film thickness to be $1.0 \mu \mathrm{m}$. For the overlayer a strong Raman-active resonance was assumed to be positioned at $900 \mathrm{~cm}^{-1}$ with an amplitude five times larger than the nonresonant contribution. For this configuration the op-

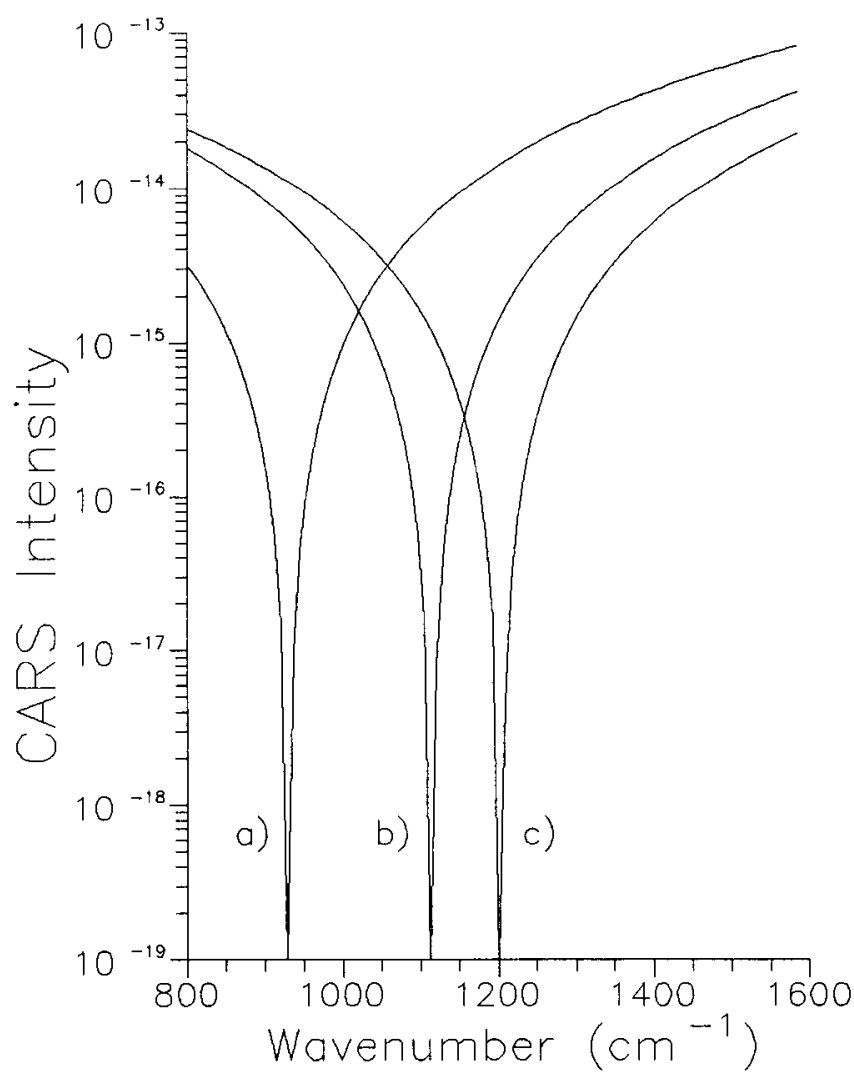

FIG. 6. Nonresonant CARS signal for the TE101 mode combination as a function of spectral shift for three different pump wavelengths. The film thickness is $1.0 \mu \mathrm{m}$. Pump wavelengths: (a) $532 \mathrm{~nm}$; (b) 500 $\mathrm{nm}$; (c) $450 \mathrm{~nm}$. 

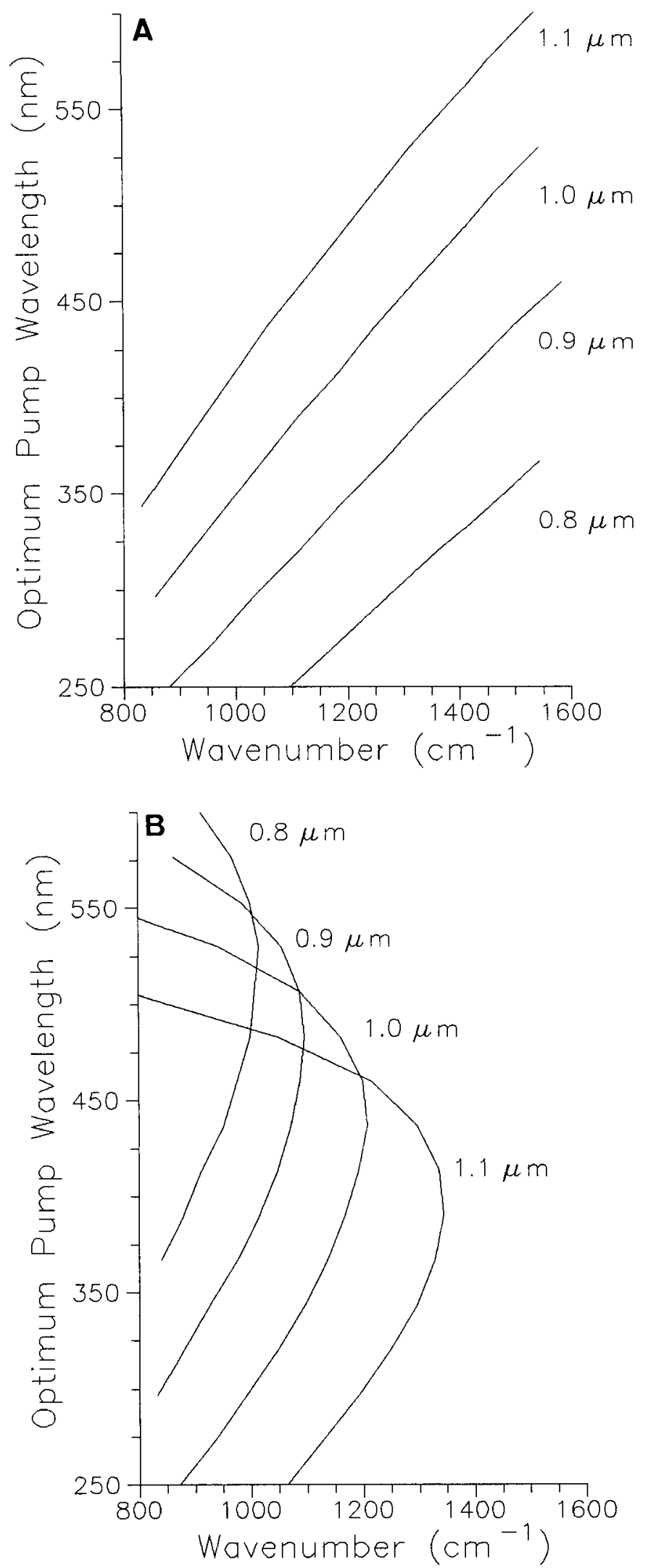

FIG. 7. Optimum pump tuning curves. (A) TE100 mode combination; (B) TE101 mode combination. The film thicknesses are indicated in each figure.

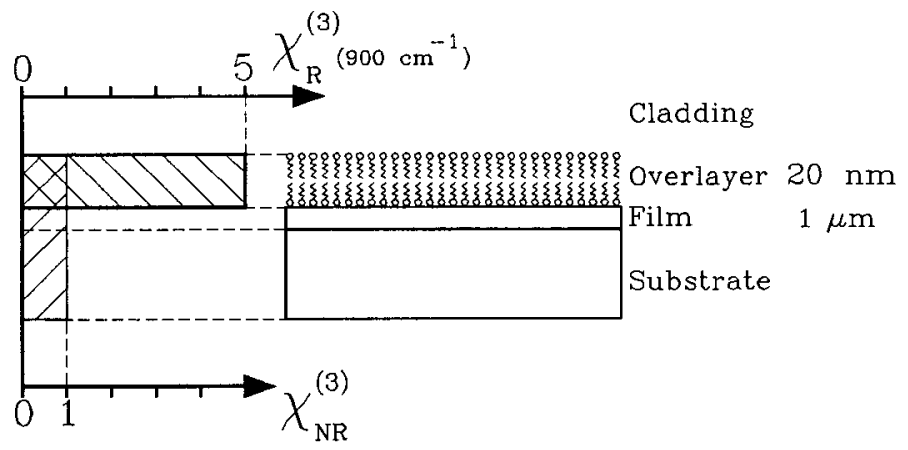

FIG. 8. Waveguide with multiple overlayers. Thicknesses, as well as nonresonant and resonant contributions of each layer, are indicated.

timum pump conditions were calculated, and the tuning curves are given in Fig. 9 together with the Stokes and CARS curves. With these tuning conditions a CARS spectrum was calculated and is given in Fig. 10a. A background-free spectrum which exhibits a peak at $900 \mathrm{~cm}^{-1}$ was obtained. In the case where the system is optimized for only one specific spectral shift (e.g., at $900 \mathrm{~cm}^{-1}$ ) and the pump wavelength is not changed during the scan, the result is given by Fig. 10b. The same resonance at $900 \mathrm{~cm}^{-1}$ can be seen, but a large background comes up at $875 \mathrm{~cm}^{-1}$ and $925 \mathrm{~cm}^{-1}$. The suppression method also enables heterodyning experiments. In order to measure a heterodyned spectrum, a controlled amount of background must be mixed with the resonant signal. In case the complete optimum wavelength combination is shifted by $+8 \mathrm{~nm}$, a small amount of background is mixed

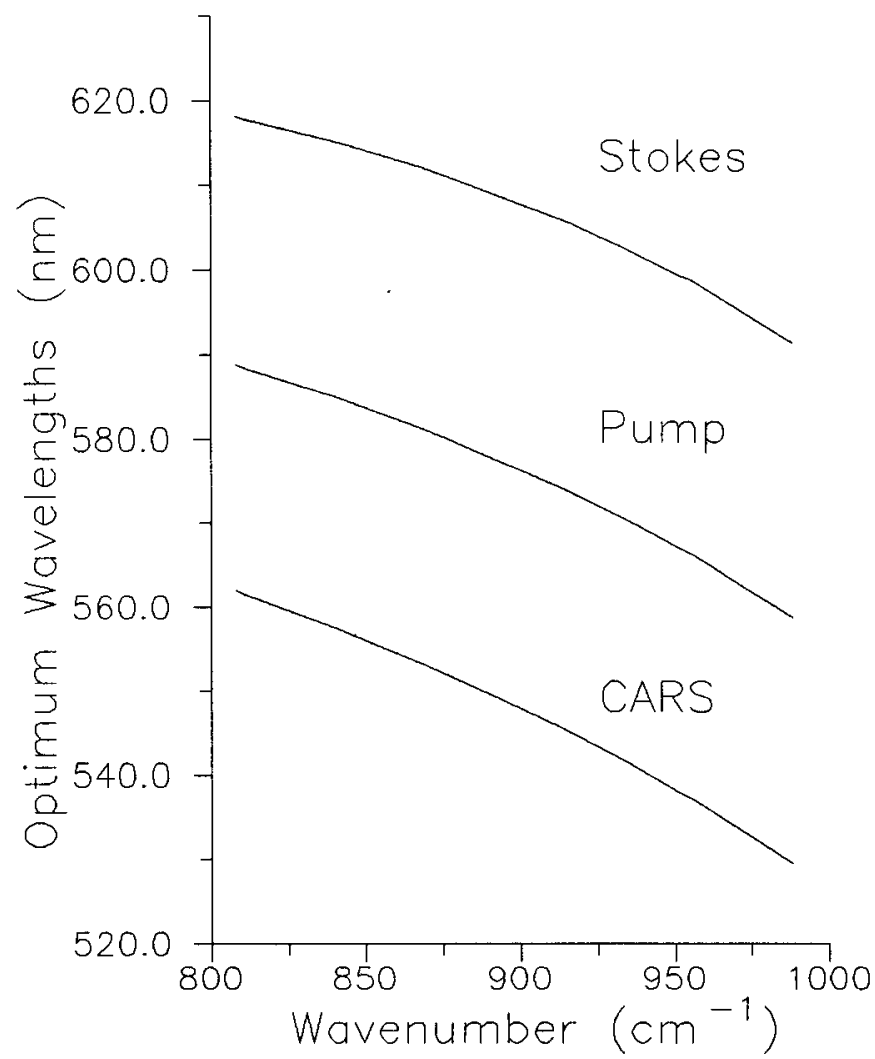

FIG. 9. TE101 Optimum pump tuning curves for waveguide with overlayer. 


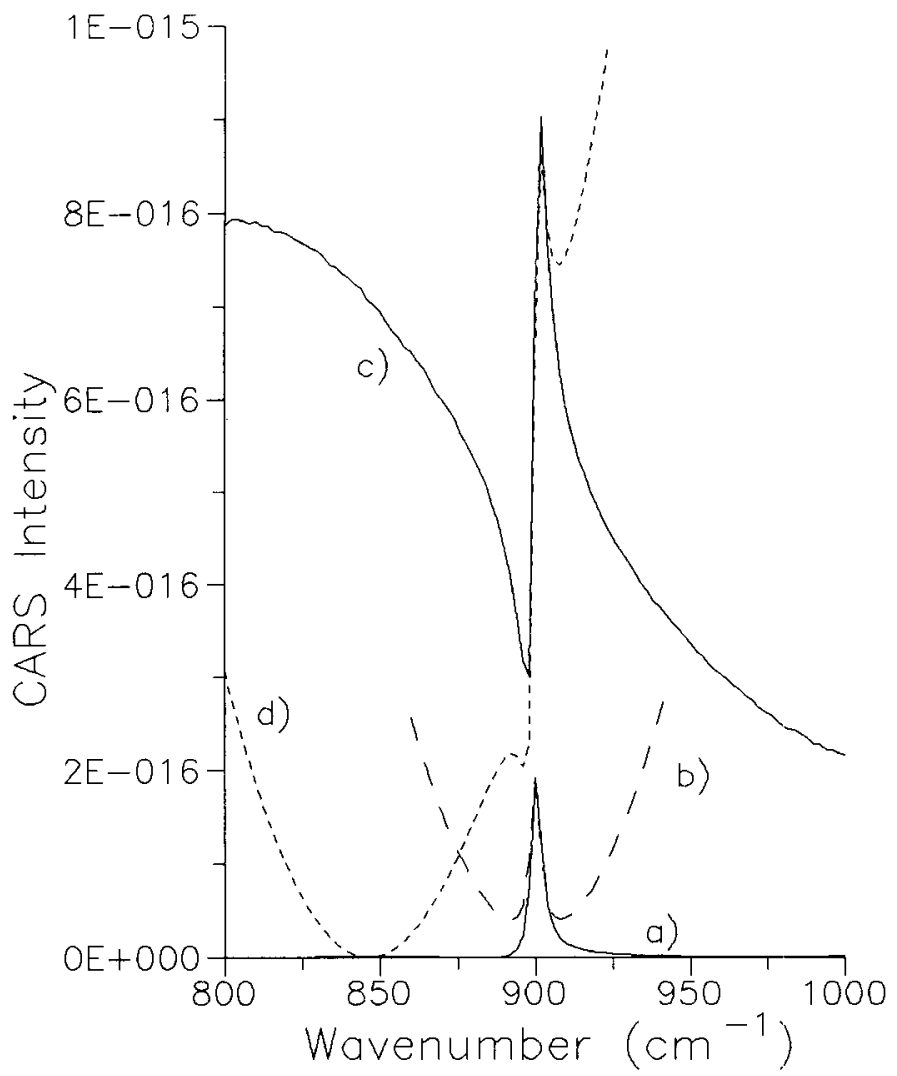

FIG. 10. TE101 CARS spectra calculated under different conditions: (a) with optimum pump tuning; (b) without optimum pump tuning (pump optimized at $900 \mathrm{~cm}^{-1}$ ); (c) heterodyned spectrum with optimum pump tuning; $(d)$ heterodyned spectrum without optimum pump tuning.

with the resonant signal and a typical heterodyned spectrum is obtained, as is given in Fig. 10c. In case no pump tuning is applied and the pump is shifted by $+8 \mathrm{~nm}$, a spectrum as given by Fig. 10d is measured. With this pump wavelength, the nonresonant suppression is now optimized around $845 \mathrm{~cm}^{-1}$, where no background is measured. However, around $900 \mathrm{~cm}^{-1}$ a strong nonconstant background interferes with the vibrational line, yielding a complex spectrum with only a small feature of the $900-\mathrm{cm}^{-1}$ vibration.

\section{CONCLUSIONS}

Waveguide CARS can be successfully applied to thinlayer dielectric films; however the large background contribution from the waveguide itself dominates the spectrum, and small surface effects due to thin layers on top of the guiding structure cannot be monitored.

The suppression of strong nonresonant CARS signals from waveguide structures is based on the destructive interference between different contributions from separate layers. By proper choice of the mode combination, optimum waveguide configuration and wavelength conditions the contributions can be made to cancel. This background-free situation is limited to a narrow spectral interval due to the spectral dependence of refractive indices and mode distribution functions. We have presented a new method to increase this spectral interval by applying a tunable pump and Stokes source. With these two tunable sources, the optimum suppression situation can be maintained over a larger spectral interval.

For a dielectric waveguide we have demonstrated these possibilities and showed a large increase. The method also allows the possibility of heterodyning the resonant signal with a small amount of nonresonant signal, thereby increasing the resonant/nonresonant interference term. This heterodyning effect gives good results only when combined with optimum pump tuning.

\section{ACKNOWLEDGMENTS}

We wish to acknowledge the support provided to one of us (W.P. de B.) by the S.T.W., Foundation for Technical Sciences, Grant TTN00.2418 .

1. J. F. Rabolt and J. D. Swalen, "Structure and Orientation in Thin Films: Raman Studies with Integrated Optical Techniques," in Spectroscopy of Surfaces, R. J. H. Clark and R. E. Hester, Eds. (Wiley, Chichester, 1988), Vol. 16, p. 1.

2. N. E. Schlotter and J. F. Rabolt, J. Phys. Chem. 88, 2062 (1984).

3. W. M. Hetherington III, N. E. Van Wyck, E. W. Koenig, G. I. Stegeman, and R. M. Fortenberry, Opt. Lett. 9, 88 (1984).

4. G. I. Stegeman, R. Fortenberry, C. Karaguleff, R. Moshrefzadeh, W. M. Hetherington III, N. E. Van Wyck, and J. E. Sipe, Opt. Lett. 8, 295 (1983).

5. D. Narayana Rao, R. Burzynski, and P. N. Prasad, Opt. Comm. 82, 357 (1991)

6. J. J. Song, G. L. Eesley, and M. D. Levenson, Appl. Phys. Lett. 29, 567 (1976).

7. J. L. Oudar, R. W. Smith, and Y. R. Shen, Appl. Phys. Lett. 34, 758 (1979).

8. W. M. Hetherington III, Z. Z. Ho, E. W. Koenig, G. I. Stegeman, and R. M. Fortenberry, Proc. SPIE 620, 102 (1986).

9. W. M. K. P. Wijekoon, Z. Z. Ho, and W. M. Hetherington III, J. Chem. Phys. 86, 4384 (1987).

10. W. M. Hetherington III, E. W. Koenig, and W. M. K. P. Wijekoon, Chem. Phys. Lett. 134, 203 (1987).

11. Z. Z. Ho, W. M. K. P. Wijekoon, E. W. Koenig, and W. M. Hetherington III, J. Chem. Phys. 91, 757 (1987).

12. W. M. Hetherington III, Z. Z. Ho, E. W. Koenig, G. I. Stegeman, and R. M. Fortenberry, Chem. Phys. Lett. 128, 150 (1986).

13. D. Marcuse, "Theory of Dielectric Optical Waveguides," in Quantum Electronics: Principles and Applications, P. L. Liao and P. L. Kelley, Eds. (Academic Press, London, 1991), 2nd ed.

14. C. A. Ward, K. Bhasin, R. J. Bell, R. W. Alexander, and I. Tyler, J. Chem. Phys. 62, 1674 (1975).

15. A. K. Ghatak, K. Thyagarajan, and M. R. Shenoy, J. Lightwave Tech. LT-5, 660 (1987).

16. J. E. Sipe and G. I. Stegeman, J. Opt Soc. Am. 69, 1676 (1979).

17. G. I. Stegeman and C. T. Seaton, J. Appl. Phys. 58, R57 (1985).

18. R. Brakel and F. W. Schneider, "Polarization CARS Spectroscopy," in Advances in Non-Linear Spectroscopy, R. J. H. Clark and R. E. Hester, Eds. (Wiley, Chichester, 1988), Vol. 15, pp. 149-192.

19. F. M. Kamga and M. G. Sceats, Opt. Lett. 5, 126 (1980). 